\title{
On the Initial Condition Problem of the Time Domain PMCHWT Surface Integral Equation
}

\author{
Ismail E. Uysal and Hakan Bagci \\ Division of Computer, Electrical and \\ Mathematical Sciences and Engineering \\ King Abdullah University of Science and Technology \\ Thuwal 23955-6900, Saudi Arabia \\ e-mails: \{ismail.uysal, hakan.bagci\} @ kaust.edu.sa
}

\author{
A. Arif Ergin and H. Arda Ulku \\ Department of Electronics Engineering \\ Gebze Technical University \\ Gebze, Kocaeli, 41400, Turkey \\ e-mails: \{aergin, haulku\}@gtu.edu.tr
}

\begin{abstract}
Non-physical, linearly increasing and constant current components are induced in marching on-in-time solution of time domain surface integral equations when initial conditions on time derivatives of (unknown) equivalent currents are not enforced properly. This problem can be remedied by solving the time integral of the surface integral for auxiliary currents that are defined to be the time derivatives of the equivalent currents. Then the equivalent currents are obtained by numerically differentiating the auxiliary ones. In this work, this approach is applied to the marching on-in-time solution of the time domain Poggio-MillerChan-Harrington-Wu-Tsai surface integral equation enforced on dispersive/plasmonic scatterers. Accuracy of the proposed method is demonstrated by a numerical example.
\end{abstract}

\section{INTRODUCTION}

Linearly increasing and constant current components are often observed in marching on-in-time (MOT) solution of time domain surface integral equations (TD-SIEs) [1]-[4]. The presence of these spurious components is attributed to the fact that initial conditions on time derivatives of unknown equivalent currents are not enforced properly [3], [4]. For example, the time domain electric field integral equation (TDEFIE) expresses the time derivative of the scattered electric field in terms of the current and its second-order time derivative. Consequently, the MOT scheme used for solving the TD-EFIE has to enforce two initial conditions, one on the current and another one on its second-order derivative. However, traditional MOT schemes assume zero initial condition only on the current without considering the possibility of discontinuous/fast changes in the current's time derivatives.

In [5], [6], an MOT scheme, which solves the time domain Poggio-Miller-Chan-Harrington-Wu-Tsai surface integral equation (TD-PMCHWT-SIE), is developed for computing fields scattered from dispersive objects. Like the TDEFIE, the linearly increasing and constant current components contaminates the MOT solution of this equation. As expected, these spurious components also affect the frequency response and might suppress physical resonance modes, especially when sensitive plasmonic interactions are considered.

In this work, the method developed in [3], is used for eliminating these spurious modes from the solution. This approach constructs the TD-PMCHWT-SIE in the time integral of the equivalent currents and uses the traditional MOT scheme to solve it for these auxiliary unknown variables. Differentiating these variables in time (during MOT steps) yields a solution that is free from spurious constant and/or linearly increasing components.

\section{FORMULATION}

Consider an object residing in an unbounded background medium. Let $\left\{\varepsilon_{0}, \mu_{0}\right\}$ and $\left\{\varepsilon_{1}(t), \mu_{1}\right\}$ represent the permittivity and permeability of the background medium and the object. Electromagnetic field $\left\{\mathbf{E}^{\text {inc }}(\mathbf{r}, t), \mathbf{H}^{\text {inc }}(\mathbf{r}, t)\right\}$ excites the object. Using the equivalence theorem and expressing the scattered field in terms of equivalent surface current densities $\mathbf{J}(\mathbf{r}, t)$ and $\mathbf{M}(\mathbf{r}, t)$ on the surface $S$ of the object, one can construct the TD-PMCHWT-SIE:

$$
\begin{aligned}
& \hat{\mathbf{n}}(\mathbf{r}) \times \partial_{t} \mathbf{E}^{\mathrm{inc}}(\mathbf{r}, t)=-\hat{\mathbf{n}}(\mathbf{r}) \times\left[\mathcal{L}_{0}\left\{\mu_{0} \mathbf{J}(\mathbf{r}, t)\right\}\right. \\
& -\mathcal{Q}_{0}\left\{\varepsilon_{0}^{-1} \mathbf{J}(\mathbf{r}, t)\right\}+\mathcal{K}_{0}\{\mathbf{M}(\mathbf{r}, t)\}+\mathcal{L}_{1}\left\{\mu_{1} \mathbf{J}(\mathbf{r}, t)\right\} \\
& \left.-\mathcal{Q}_{1}\left\{\bar{\varepsilon}_{1}(t) * \mathbf{J}(\mathbf{r}, t)\right\}+\mathcal{K}_{1}\{\mathbf{M}(\mathbf{r}, t)\}\right] \\
& \hat{\mathbf{n}}(\mathbf{r}) \times \partial_{t} \mathbf{H}^{\mathrm{inc}}(\mathbf{r}, t)=-\hat{\mathbf{n}}(\mathbf{r}) \times\left[\mathcal{L}_{0}\left\{\varepsilon_{0}^{-1} \mathbf{M}(\mathbf{r}, t)\right\}\right. \\
& -\mathcal{Q}_{0}\left\{\mu_{0}^{-1} \mathbf{M}(\mathbf{r}, t)\right\}-\mathcal{K}_{0}\{\mathbf{J}(\mathbf{r}, t)\}+\mathcal{L}_{1}\left\{\varepsilon_{1}(t) * \mathbf{M}(\mathbf{r}, t)\right\} \\
& \left.-\mathcal{Q}_{1}\left\{\mu_{1}^{-1} \mathbf{M}(\mathbf{r}, t)\right\}-\mathcal{K}_{1}\{\mathbf{J}(\mathbf{r}, t)\}\right] .
\end{aligned}
$$

Here, $\hat{\mathbf{n}}(\mathbf{r})$ is the unit normal vector on $S, \bar{\varepsilon}_{1}(t)$ is the inverse permittivity function, and

$$
\begin{aligned}
& \mathcal{L}_{m}\{\mathbf{X}(\mathbf{r}, t)\}=\int_{S} G_{m}(R, t) * \partial_{t}^{2} \mathbf{X}\left(\mathbf{r}^{\prime}, t\right) d s^{\prime} \\
& \mathcal{Q}_{m}\{\mathbf{X}(\mathbf{r}, t)\}=\nabla \int_{S} G_{m}(R, t) * \nabla^{\prime} \cdot \mathbf{X}\left(\mathbf{r}^{\prime}, t\right) d s^{\prime} \\
& \mathcal{K}_{m}\{\mathbf{X}(\mathbf{r}, t)\}=\nabla \times \int_{S} G_{m}(R, t) * \partial_{t} \mathbf{X}\left(\mathbf{r}^{\prime}, t\right) d s^{\prime}
\end{aligned}
$$

for $m \in\{0,1\}$. In (3), $R=\left|\mathbf{r}-\mathbf{r}^{\prime}\right|$ and $G_{m}(R, t)$ is Green function of the unbounded medium with $\varepsilon_{m}$ and $\mu_{m}$. The MOT scheme developed in [5], [6] can be used to solve (1) for unknowns $\mathbf{J}(\mathbf{r}, t)$ and $\mathbf{M}(\mathbf{r}, t)$.

TD-PMCHWT-SIE in (1)-(2) involves the second-order time derivative of equivalent surface current densities due to $\mathcal{L}_{m}\{\cdot\}$ operator, which results in non-physical linearly increasing and constant components in the MOT solution as explained above. These components can be eliminated through the use of 
auxiliary surface current densities $\mathbf{P}(\mathbf{r}, t)$ and $\mathbf{Q}(\mathbf{r}, t)$ defined as

$$
\partial_{t}^{2} \mathbf{P}(\mathbf{r}, t)=\mathbf{J}(\mathbf{r}, t), \partial_{t}^{2} \mathbf{Q}(\mathbf{r}, t)=\mathbf{M}(\mathbf{r}, t) .
$$

Inserting (3) into (1) and integrating the resulting equation in time twice yield the TD-PMCHWT-SIE in unknown $\mathbf{P}(\mathbf{r}, t)$ and $\mathbf{Q}(\mathbf{r}, t)$ :

$$
\begin{aligned}
& \hat{\mathbf{n}}(\mathbf{r}) \times \partial_{t}^{-1} \mathbf{E}^{\mathrm{inc}}(\mathbf{r}, t)=-\hat{\mathbf{n}}(\mathbf{r}) \times\left[\mathcal{L}_{0}\left\{\mu_{0} \mathbf{P}(\mathbf{r}, t)\right\}\right. \\
& -\mathcal{Q}_{0}\left\{\varepsilon_{0}^{-1} \mathbf{P}(\mathbf{r}, t)\right\}+\mathcal{K}_{0}\{\mathbf{Q}(\mathbf{r}, t)\}+\mathcal{L}_{1}\left\{\mu_{1} \mathbf{P}(\mathbf{r}, t)\right\} \\
& \left.-\mathcal{Q}_{1}\left\{\bar{\varepsilon}_{1}(t) * \mathbf{P}(\mathbf{r}, t)\right\}+\mathcal{K}_{1}\{\mathbf{Q}(\mathbf{r}, t)\}\right] \\
& \hat{\mathbf{n}}(\mathbf{r}) \times \partial_{t}^{-1} \mathbf{H}^{\mathrm{inc}}(\mathbf{r}, t)=-\hat{\mathbf{n}}(\mathbf{r}) \times\left[\mathcal{L}_{0}\left\{\varepsilon_{0}(t) * \mathbf{Q}(\mathbf{r}, t)\right\}\right. \\
& -\mathcal{Q}_{0}\left\{\mu_{0}^{-1} \mathbf{Q}(\mathbf{r}, t)\right\}-\mathcal{K}_{0}\{\mathbf{P}(\mathbf{r}, t)\}+\mathcal{L}_{1}\left\{\varepsilon_{1}(t) * \mathbf{Q}(\mathbf{r}, t)\right\} \\
& \left.-\mathcal{Q}_{1}\left\{\mu_{1}^{-1} \mathbf{Q}(\mathbf{r}, t)\right\}-\mathcal{K}_{1}\{\mathbf{P}(\mathbf{r}, t)\}\right] .
\end{aligned}
$$

Here, $\partial_{t}^{-1}$ denotes integration in time. To numerically solve (4), first $\mathbf{P}(\mathbf{r}, t)$ and $\mathbf{Q}(\mathbf{r}, t)$ are approximated using RaoWilton-Glisson functions $\mathbf{f}_{n}(\mathbf{r})$ and Lagrange interpolation functions $T(t)$ as

$$
\begin{aligned}
& \mathbf{P}(\mathbf{r}, t)=\sum_{j=1}^{N_{t}} \sum_{n=1}^{N_{s}} P_{j n} T(t-j \Delta t) \mathbf{f}_{n}(\mathbf{r}) \\
& \mathbf{Q}(\mathbf{r}, t)=\sum_{j=1}^{N_{t}} \sum_{n=1}^{N_{s}} Q_{j n} T(t-j \Delta t) \mathbf{f}_{n}(\mathbf{r})
\end{aligned}
$$

where $\Delta t$ is time step size and $P_{j n}$ and $Q_{j n}$ are the unknown current coefficients. Inserting (5) into (4) and Galerkin testing the resulting equations at discrete times $i \Delta t$ yield the MOT matrix system:

$$
\mathbf{Z}_{0} \mathbf{I}_{i}=\mathbf{V}_{i}-\sum_{j=1}^{i-1} \mathbf{Z}_{i-j} \mathbf{I}_{j}, i=1, . ., N_{t} .
$$

Here, $\mathbf{I}_{i}$ and $\mathbf{V}_{i}$ store the unknown coefficients and the tested incident fields, respectively, and $\mathbf{Z}_{i-j}$ are the MOT matrices. $\mathbf{I}_{i}, i=1, . ., N_{t}$ are obtained in an iterative fashion via time marching. Then, coefficients of $\mathbf{J}(\mathbf{r}, t)$ and $\mathbf{M}(\mathbf{r}, t)$ are computed from $P_{j n}$ and $Q_{j n}$ using numerical differentiation. It should be noted here that (i) $\mathbf{Z}_{i-j}$ are same as the matrices obtained from the MOT discretization of (1) and computed using the numerical scheme described in [5], [6], and (ii) $\mathbf{P}(\mathbf{r}, t)$ and $\mathbf{Q}(\mathbf{r}, t)$ obtained by solving (6) are contaminated by spurious components. However these are eliminated from $\mathbf{J}(\mathbf{r}, t)$ and $\mathbf{M}(\mathbf{r}, t)$ by the numerical differentiation applied to $\mathbf{P}(\mathbf{r}, t)$ and $\mathbf{Q}(\mathbf{r}, t)$ [see (3)]. The numerical differentiation is implemented using a finite difference scheme whose order is larger than or equal to the order of $T(t)$ not to introduce additional errors.

\section{NumericAl Results}

Transient fields scattered from a gold sphere are computed by two MOT schemes solving (1) and (4). The sphere has a radius of $50 \mathrm{~nm}$ and is centered at the origin in free space. Time domain permittivity and Green function associated with gold are obtained using the method described in [6]. The electric field of the incident plane wave $\mathbf{E}^{\text {inc }}(\mathbf{r}, t)=$ $\hat{\mathbf{x}} \cos \left(2 \pi f_{0} \bar{t}\right) \exp \left(-\bar{t}^{2} / \sigma^{2}\right)$, where $\bar{t}=t-t_{0}-\hat{\mathbf{z}} \cdot \mathbf{r} \sqrt{\varepsilon_{0} \mu_{0}}$, $t_{0}=3.5 \sigma, \sigma=3 /\left(2 \pi f_{\mathrm{bw}}\right), f_{\mathrm{bw}}=600 \mathrm{THz}$, and $f_{0}=900$ THz. Unknowns are discretized using $N_{s}=714$ RWG basis

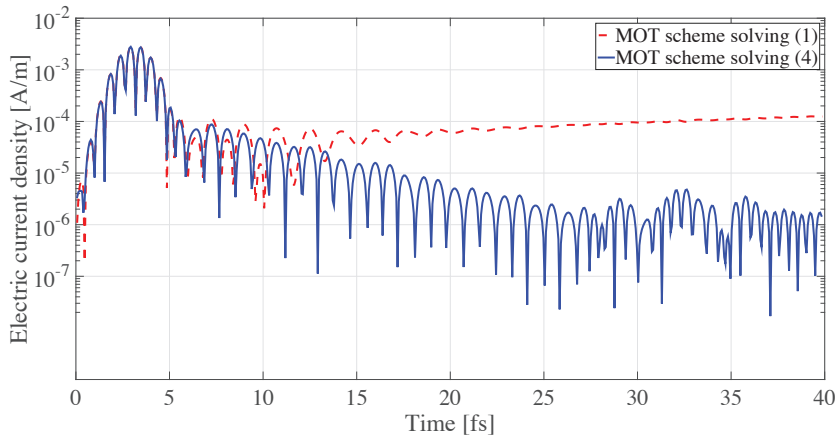

Fig. 1. One of the coefficients of the electric current density expansion computed by the two MOT schemes solving (1) and (4).

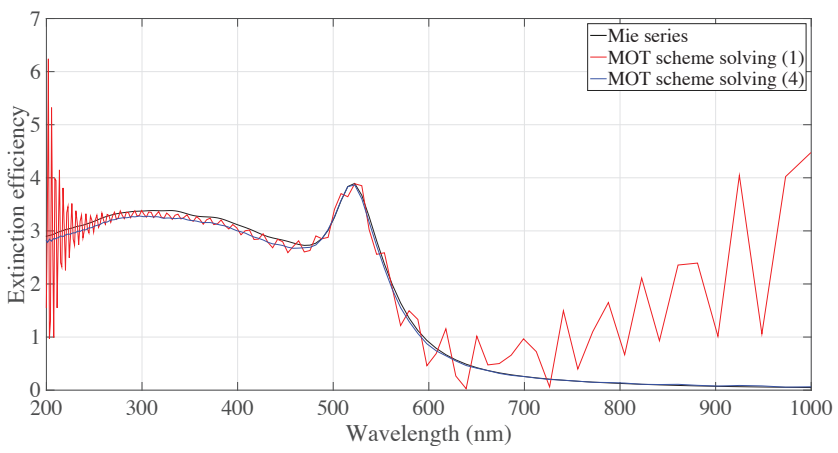

Fig. 2. Extinction cross sections obtained from Fourier transformed electric and magnetic surface current densities, which are computed by the two MOT schemes.

functions and simulations are carried out for $N_{t}=600$ time steps with $\Delta t=0.066$ fs.

Fig. 1 compares one of the coefficients of the electric current density expansion computed by the MOT schemes by solving (1) and (4). It is clear the latter result is free from linearly increasing and constant components. Fig. 2 compares extinction cross sections which are obtained from Fourier transformed electric and magnetic surface current densities that are computed by the two MOT schemes. It is clearly shown that the solution free from spurious components produces more accurate frequency domain results.

\section{REFERENCES}

[1] D. S. Weile, G. Pisharody, N.-W. Chen, B. Shanker, and E. Michielssen, "A novel scheme for the solution of the time-domain integral equations of electromagnetics," IEEE Trans. Antennas Propag., vol. 52, pp. 283-295, 2004.

[2] Y. Shi, H. Bagci and M. Lu, "On the internal resonant modes in marchingon-in-time solution of the time domain electric field integral equation," IEEE Trans. Antennas. Propag., vol. 61, no. 8, pp. 4389-4392, 2013.

[3] H. A. Ulku and A. A. Ergin, "Solution of the initial condition problem of the time-domain EFIE," Proc. Comput. Electromagn. Workshop (CEM), pp. 17-18, 2013.

[4] Y. Shi, H. Bagci and M. Lu, "On the static loop modes in the marchingon-in-time solution of the time-domain electric field integral equation," IEEE Antennas Wireless Propag. Lett., vol. 13, pp. 317-320, 2014.

[5] I. E. Uysal, H. A. Ulku, and H. Bagci, "MOT solution of the PMCHWT equation for analyzing transient scattering from conductive dielectrics," IEEE Antennas Wireless Propag. Lett., vol. 14, pp. 507-510, 2015.

[6] I. E. Uysal, H. A. Ulku, and H. Bagci, "Transient analysis of electromagnetic wave interactions on plasmonic nanostructures using a surface integral equation solver," J. Opt. Soc. Am. A, vol. 33, no. 9, pp. 17471759, 2016. 